\title{
NOTAS TAXONÓMICAS SOBRE EL GÉNERO ONONIS L. (FABACEAE) PARA LA FLORA DE MARRUECOS
}

\author{
Juan Antonio DEVESA ALCARAZ
}

RESUMEN. Notas taxonómicas sobre el género Ononis L. (Fabaceae) para la flora de Marruecos. Se describen dos nuevas especies de Ononis para el norte de África, O.cuatrecasasii y O.gines-lopezii, y se confirma la validez taxonómica de $O$.jahandiezii Maire \& Weiller. Se elaboran claves para la segregación de $O$. penduliflora dentro del complejo de $O$. pendula.

Palabras clave. Angiospermae, Leguminosae, Ononis, Taxonomía, Marruecos.

ABSTRACT. Taxonomic notes about genus Ononis L. (Fabaceae) for the Morocco flora. Two new species of Ononis from the northwest of Morocco are described: $O$. cuatrecasasii and $O$. gines-lopezii. The taxonomic validity of $O$. jahandiezii Maire \& Weiller is verified. A dichotomic key for the segregation of $O$. penduliflora within the $O$. pendula complex is given.

Key words. Angiospermae, Leguminosae, Ononis, Taxonomy, Morocco.

\section{INTRODUCCIÓN}

El género Ononis L. está distribuido fundamentalmente por la Región Mediterránea (alcanza la Región Macaronésica y Asia), comprende alrededor de unas 70 especies (Sirjaev, 1932) y presenta su máxima representación en el W de la Región Mediterránea, en particular en el SW de España y NW de África.

Su conocimiento taxonómico se debe fundamentalmente a Sirjaev (1932), el monógrafo del género, si bien existen contribuciones taxonómicas de importancia regional o local, particularmente para la
Península Ibérica (Losa, 1958; Ivimey-Cook, 1968; Ladero \& Socorro, 1982; López, 1985; Devesa, 1986, 1988 a \& b, y 1992, etc.).

La preparación por el autor de la síntesis taxonómica del género Ononis para la Checklist of Vascular Plants of Northern Morocco, ha puesto de manifiesto una nutrida representación del género en el extremo $\mathrm{N}$ de Marruecos, donde están representadas 49 especies (no menos de 58 taxones), de algunas de las cuales se dudaba sobre su validez taxonómica $(O$. jahandiezii Maire \& Weiller), había dificultad de comprensión (O. penduliflora) y otras, en particular, son nuevas especies $(O$. cuatrecasasii y O. gines-lopezii) que se describen a continuación. 


\section{RESULTADOS}

\section{Ononis cuatrecasasii Devesa, sp.nov.}

Planta annua, ad basem valde ramosa. Caulibus ascendentibus, usque ad $30 \mathrm{~cm}$, pilis pluricelularibus $0,5 \mathrm{~mm}$ vel longioribus, praecipue eglandulosis, vestitis. Foliis caulinis trifoliatis, foliolis obovatis vel obovato-cuneatis, perspicue dentatis, pilis glandulosis brevibus pluricelularibus instructis, floralibus unifoliolatis, foliolo obovato, truncato, saltem ad apicem perspicue dentato; stipulis petiolo adnatis, dentatis apice libero, pilis glandulosis brevibus pluricelularibus instructis. Racimo laxiusculo, floribus breviter pedicellatis. Calyce 8-11 mm, pilis pluricelularibus glandulosis $0,5 \mathrm{~mm}$ vel brevioribus praecipue ad dentes instructo; dentibus triangulari-lanceolatis tubo 2 3 plo longioribus. Corolla 6-9 mm, calyce breviora, vexillo roseo, puberulento-glanduloso. Legumine 4-6 $\mathrm{mm}$, ovoideo, pilis pluricelularibus $0,5 \mathrm{~mm}$ vel brevioribus praecipue ad apicem instructo. Seminibus 1,7-2,3 mm, laevibus, fuscis vel olivaceis.

Anual, muy ramificada en la base. Tallos de hasta $30 \mathrm{~cm}$, ascendentes, con pelos pluricelulares de más de $0,5 \mathrm{~mm}$, en su mayoría eglandulares. Hojas caulinares trifoliadas, con folíolos obovados u obovado-cuneados, conspícuamente dentados, con pelos pluricelulares glandulosos cortos; hojas de la inflorescencia unifoliadas, con folíolo obovado, truncado y conspícuamente dentado al menos distalmente. Estípulas parcialmente soldadas al pecíolo, dentadas, con pelos pluricelulares glandulosos cortos. Inflorescencia en racimo laxo, con flores cortamente pediceladas. Cáliz de 8-11 $\mathrm{mm}$, con pelos pluricelulares glandulosos de menos de $0,5 \mathrm{~mm}$ y concentrados sobre todo en los dientes; dientes calicinales triangular-lanceolados, 2-3 veces tan largos como el tubo. Corola de 6-9 mm, menor que el cáliz, con estandarte rosado, puberulentoglanduloso. Legumbre de 4-6 mm, ovoidea, con pelos pluricelulares glandulosos de menos de 0,5 mm concentrados sobre todo en el ápice. Semillas de 1,7-2,3 mm, lisas, marrones u oliváceas (fig. 1).

Dedicada al Dr. J. Cuatrecasas, recientemente fallecido.

Especie muy similar a O. cossoniana Boiss. \&
Reuter, de la que difiere por presentar al menos las hojas distales de la inflorescencia unifoliadas, con folíolos obovados y conspícuamente dentados distalmente; sus flores con corolas de 6-9 mm, más cortas que los cálices, y sus semillas menores, de 1,7-2,3 mm.

Holotypus. - MARRUECOS. «826803 (impr.) (a) (manuscr.) / PLANTAE MAROCCANAE ANNO 1992 LECTAE (impr.) / Javier FERNANDEZ CASAS \& Julián MOLERO (impr.) / FABACEAE (impr.)/Ononis (impr.) cuatrecasasii Devesa, sp. nov. (manuscr.) / KENITRA: prope Souk et Tnine Jorf-el-Mellah, (impr.) / 30STD61, inter segetes, substrato calcareo, Fernán- (impr.) / dez Casas 13786 \& Molero, 7-V-1992. (impr.) / Det. Devesa (manuscr.) / ¡HOLOTYPUS! (impr.)» (BC 826803 (A)).

Isotypus. - Ibídem, BC 826803 (B) \& SEV s/n !.

La especic se incluye por sus características dentro de la sección. Diffusae. ( Sirjaer, 1932:618), y más concretamente por presentar sus semillas lisas en la serie Cossonianae ( Sirjaer, 1932:629), carácter que comparte con el resto de los representantes conocidos de la serie: $O$. cossoniana Boiss. \& Reuter, O. hirta Desf. y O. cephalantha Pomel.

De $O$. cephalantha y $O$. hirta difiere, entre otros caracteres, por carecer de pelos eglandulares en el cáliz, y de O.cossoniana por sus semillas más pequeñas, de 1,7-2,3 mm (y no de (2,2-) 2,7-3,6 $\mathrm{mm}$ ), por sus corolas menores que los cálices y sus legumbres más pequeñas, de 4-5 mm (y no de 5-7 $\mathrm{mm})$ y, sobre todo, por presentar las hojas de la inflorescencia con un solo folíolo conspicuo, mientras que en O.cossoniana éstas se encuentran reducidas a las estípulas o bien sólo las más bajas presentan un folíolo terminal linear o estrechamente elíptico.

\section{Ononis gines-lopezii Devesa, sp. nov.}

Planta annua, ad basem valde ramosa. Caulibus decumbentibus, usque ad $15 \mathrm{~cm}$, pilis pluricelularibus sparsis glandulosis et eglandulosis vestitis. Foliis caulinis trifoliolatis, foliolis obovatis 


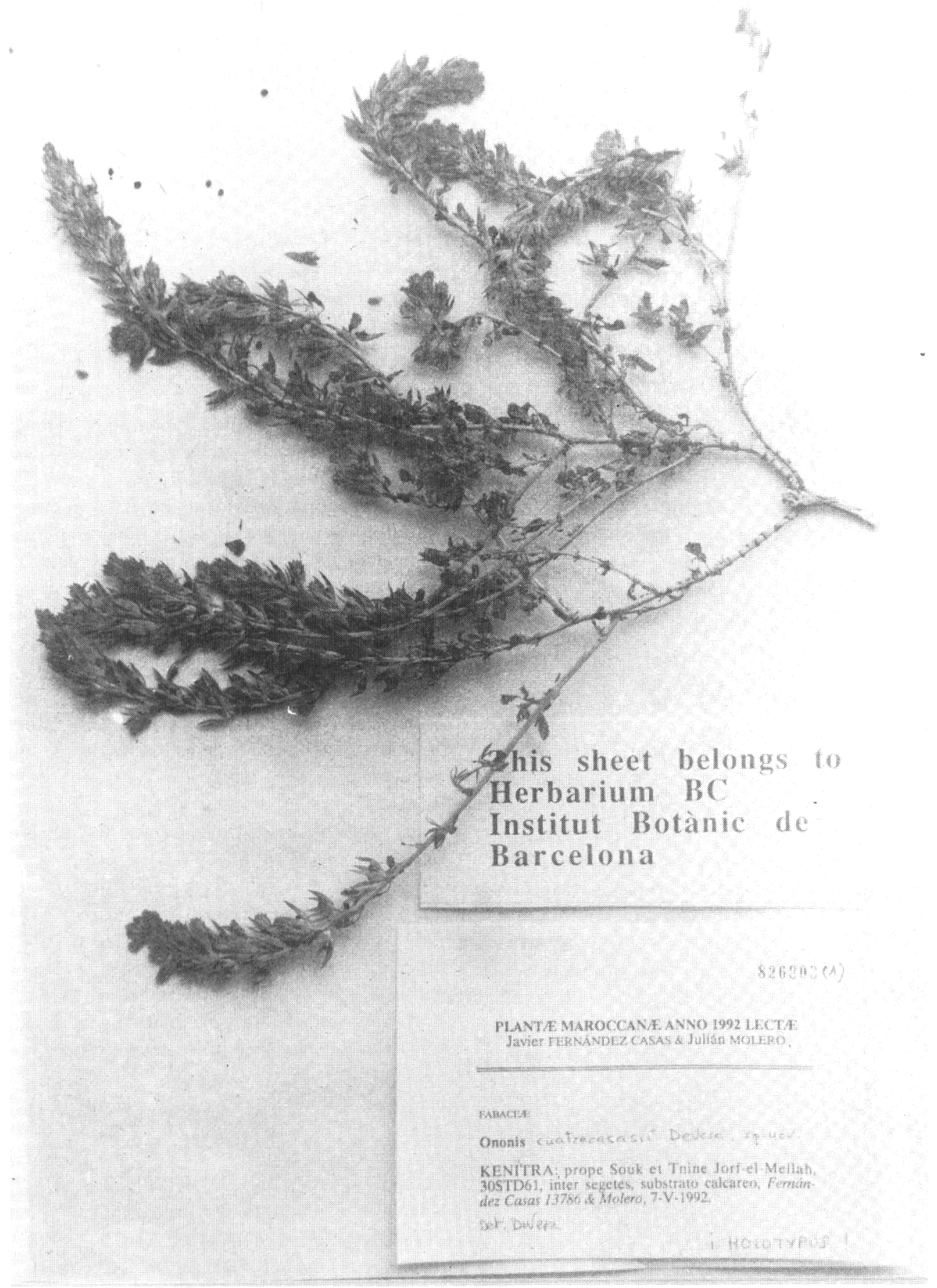

Figura 1.- Holótipo de Ononis cuatrecasasii Devesa. Holotype of Ononis cuatrecasasii Devesa. 
vel obovato-cuneatis, crassiusculis, perspique dentatis, glabrescentibus vel pilis glandulosis brevibus laxe instructis, floralibus unifoliolatis; stipulis petiolo adnatis, parte libera ovali, perspicue dentata. Racimo plus minusve laxo, floribus breviter pedicellatis. Calyce 6-8 $\mathrm{mm}$, campanulato, pilis pluricelularibus brevibus laxiusculis glandulosis et eglandulosis, ad dentes copiosoribus, instructo; tubo 3,5-4 mm; dentibuis 4-4,5 $\mathrm{mm}$, triangularilanceolatis, tubo paulo longioribus. Corolla 9-10 $\mathrm{mm}$, calyce 1,5 plo longiora, vexillo luteo, perspicue pubescente. Legumine 6-8 mm, ovoideo, leviter pubescente-glanduloso, calyce aequante vel superante. Seminibus 0,8-1,2 mm, subreniformibus, tuberculatis, fusco-purpurescentibus.

Anual, muy ramificada en la base. Tallos de hasta $15 \mathrm{~cm}$, decumbentes, con pelos pluricelulares glandulosos y eglandulares dispersos. Hojas caulinares trifoliadas, con folíolos obovados u obovado-cuneados, crasiúsculos, marcadamente dentados, glabrescentes o muy laxamente cubiertos con pelos glandulares cortos; hojas de la inflorescencia unifoliadas. Estípulas parcialmente soldadas al pecíolo, con parte libre oval, conspícuamente dentada. Inflorescencia en racimo más o menos laxo, con flores cortamente pediceladas. Cáliz de 6-8 $\mathrm{mm}$, campanulado, con pelos pluricelulares glandulosos y eglandulares, cortos y laxamente dispuestos aunque algo más abundantes en los dientes; tubo de 3,5-4 $\mathrm{mm}$; dientes calicinales de 4-4,5 mm, triangular-lanceolados. Corola de 9-10 mm, 1,5 veces mayor que el cáliz, con estandarte amarillo y marcadamente pubescente. Legumbre de 6-8 mm, ovoidea, ligeramente pubescente-glandulosa, subigual o algo más larga que el cáliz. Semillas de 0,8-1,2 mm, subreniformes, marrón-rojizas, tuberculadas (fig. 2).

\section{Dedicada al Dr. Ginés López.}

Especie muy parecida a O.variegata L. en el hábito y la ecología pero que se diferencia de esta por presentar hojas caulinares trifoliadas y no unifoliadas, así como semillas menores, de 0,8-1,2 $\mathrm{mm}$, tuberculadas y no lisas.

Holotypus.- MARRUECOS: a. «831733 (impr.) / PLANTES D'ESPAGNE (impr.) / Ononis variegata L. (s.1.) (manuscr.) / Maroc: Cabo Tres Forcas (manuscr.) / pied du Faro. (manuscr.) / 193 (manuscr.) 5-17-IV (manuscr.) F.SENNEN (impr.) / et Mauricio (manuscr.)». b. «REVISION PARA «FLORA IBERICA» (impr.)/ Ononis gines-lopezii Devesa, sp. nov. (manuscr.) / Det.rev. (impr.) Devesa (manuscr.) / VI (manuscr.) 19 (impr.) 96 (manuscr.)». (BC 831733). El holótipo designado es el ejemplar central del pliego BC 831733.

Isotypus.- BC 831732 / 831737, SEV s/n!.

Aunque identificado originalmente como O.variegata L. por Sennen \& Mauricio, el taxón difiere claramente de aquel por presentar hojas caulinares trifoliadas y semillas tuberculadas.

El taxón se incluye en la sección Diffusae (Sirjaev, 1932:618), y más concretamente en la serie Tuberculatae (Sirjaev, loc.cit.). Guarda gran parecido en hábito y ecología con O.variegata L., pero a diferencia de este presenta hojas caulinares trifoliadas y unifoliadas en la inflorescencia, así como semillas tuberculadas, mientras que O.variegata presenta hojas caulinares unifoliadas y semillas lisas y de mayor tamaño (de c. 1,5 mm). Ambas características aproximan a esta especie a O.tournefortii Cosson pero, a diferencia de este, presenta -entre otros caracteres diferenciales- cálices con pelos glandulares y eglandulares, y no solo glandulares

Ononis penduliflora Pau in Font Quer, Iter. Marocc. 1930 exsicc. 325 (1932)

Lectotypus.-. 61053 «DR. FONT QUER.- ITER MAROCCANUM, 1930 (impr.) / 325. ONONIS PENDULIFLORA Pau, nova sp. (impr.) / Hab. in arenosis, pr. El Araix; 16 aprilis (impr.) / Descr.: Ab affinibus differt corola maxima, calycis laciniis / longue lanceolatis, tubo basi attenuato (impr.) / » (MA 61053).

Isolectypus.- Ibídem, MA 61053 (2); BC 98130 (182).

Material estudiado.- MARRUECOS. Costa Atlántica. Dunes de l'Araix, $35^{\circ} 13^{\prime}-6^{\circ} 09^{\prime}$, 16.IV.1930, P.Font Quer (BC 810359). Rabat, coteaux herbeux, 14.IV.1924, E.Jahandiez PI.Maroc. exsicc. 43 (MA 61583). Larache, VI.1923, A.Caballero (MA 61586). El Guedira, c.El Araix, 


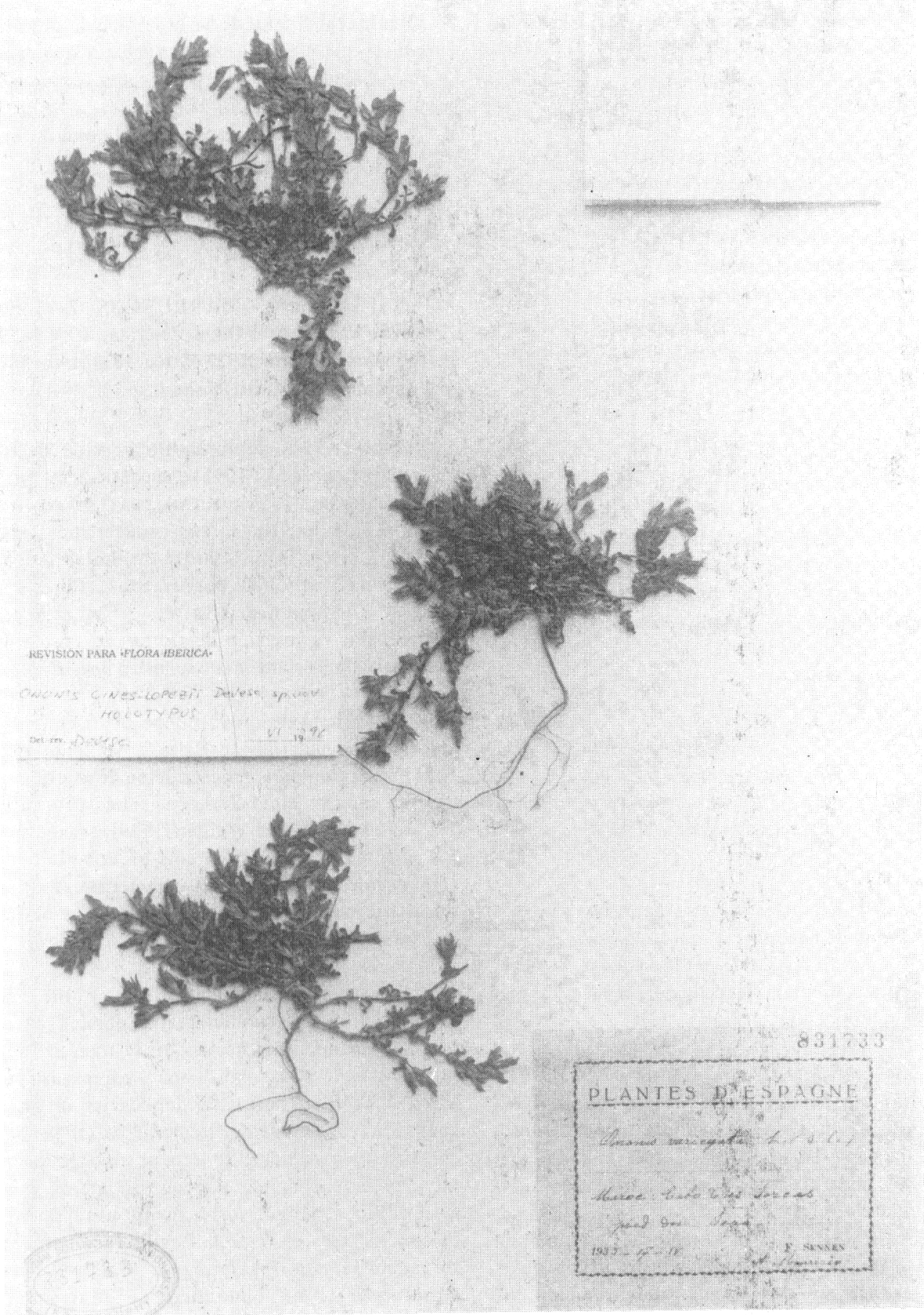

Figura 2.- Holótipo de Ononis gines-lopezii Devesa. Holotype of Ononis gines-lopezii Devesa.. 
in arenosis, inter Chamaerops, 26.IV.1930, P.Font Quer (BC 88028). Hab. in arenosis, pr. El Araix, 16.IV.1930, P.Font Quer Iter Maroc. 1930 exsicc. 325 (MA 61053 BC 98130).

O.penduliflora es un taxón afín a O.pendula Desf., especie que en el NW de Marruecos está representada por las subespecies pendula, boissieri (Sirj.) Devesa (Devesa, 1986:144) y munbyi (Sirj.) Greuter \& Burdet (Greuter \& Raus,1987: 446), siendo este último taxón (que Greuter \& cols.(1989), asimilaron a $O$.penduliflora) el que más fácilmente puede segregarse del complejo por sus dientes calicinales lineares, de menos de $0.5(-0.7) \mathrm{mm}$ de anchura.

Por las dimensiones de la corola los individuos de O.penduliflora se asemejan sobre todo a los de O.pendula subsp. boissieri, pero a diferencia de estos muestran dientes calicinales más estrechos y folíolos de las hojas generalmente oblongo-elípticos, y no obovado-cuneados como suele presentarlos este. Además, el areal de este taxón se circunscribe hasta la fecha a enclaves arenosos de la región de Larache, en tanto que O.pendula subsp. boissieri está más ampliamente distribuido en el NW de Marruecos y el SW de España.

Los taxones del complejo O.pendulaO.penduliflora pueden segregarse de la siguiente manera:

1. Dientes calicinales lineares, de menos de 0,5 ($0,7) \mathrm{mm}$ de anchura

O.pendula subsp. munbyi

I'. Dientes calicinales lanceolados, de más de $1 \mathrm{~mm}$ de anchura

2. Cáliz de 6-9 (-10) mm. Corola de (8-) 9-12($14,5) \mathrm{mm}$, hasta 1,5 veces la longitud del cáliz

O.pendula subsp. pendula

2'. Cáliz de (9,5-) 10-15 mm. Corola de 15-20 mm, 1,5-2 veces tan larga como el cáliz 3

3. Dientes calicinales a menudo de $1,5-2 \mathrm{~mm}$ de anchura, pubescente-glandulosos y con pelos eglandulares más largos sobre todo en los márgenes. Folíolos generalmente obovadocuneados

O.pendula subsp. boissieri
3'. Dientes calicinales de hasta 1,5 $\mathrm{mm}$ de anchura, pubescente-glandulosos y con pelos eglandulares muy abundantes tanto en márgenes como en el dorso. Folíolos generalmente oblongo-elípticos

O.penduliflora

Ononis jahandiezii Maire \& Weiller in Maire, Bull. Soc. Hist. Nat. Afrique N. 22: 41 (1931)

Material estudiado.- MARRUECOS. Zerhoun Zerhoun, Vallée de l'Oued Zeggota, 10.V.1929, E.Jahandiez Pl.Maroc. 1929 exsicc. 183 (MA 61961, sub «Ononis jahandiezii Maire n.sp.»).

En el herbario del Jardín Botánico de Madrid existe un pliego (MA 61961) que contiene un único ejemplar que ha sido identificado con O.jahandiezii Maire \& Weiller, siendo sus características más sobresalientes la posesión de hojas de la inflorescencia unifoliadas, superando las flores, con folíolo anchamente elíptico y largamente peciolulado; la posesión de flores con corola de estandarte pubescente y sus semillas lisas (fig. 3).

Probablemente dicho pliego se corresponde con el mismo material que Maire \& Weiller (Maire, 1931) utilizaron para la descripción de la especie: «Maroc central: Mont Zerhoun, vallée de l'Oued Zegotta (Jahandiez et Weiller, 1929)», taxón que los autores relacionaron con el complejo de O.alocecuroides, si bien las características de su estandarte lo harían excepcional dentro de la sección Salzmannianae Sirj.

La especie fue incluida por Sirjaev (1932) en la addenda final de su monografía sobre el género Ononis, sin más indicación que la transcripción literal de parte de las indicaciones expresadas por Maire \& Weiller (1.c.) en la publicación original, aunque mucho más tarde Greuter et al. (1989) han cuestionado su validez. Se trata de un buen taxón, fácilmente reconocible por sus caracteres y no asimilable a ningún otro taxón previamente reconocido para el W de la Región Mediterránea. De acuerdo con Jahandiez \& Maire (1932), O.jahandiezii es endémico de Marruecos, conociéndose hasta la fecha únicamente de la localidad clásica. 


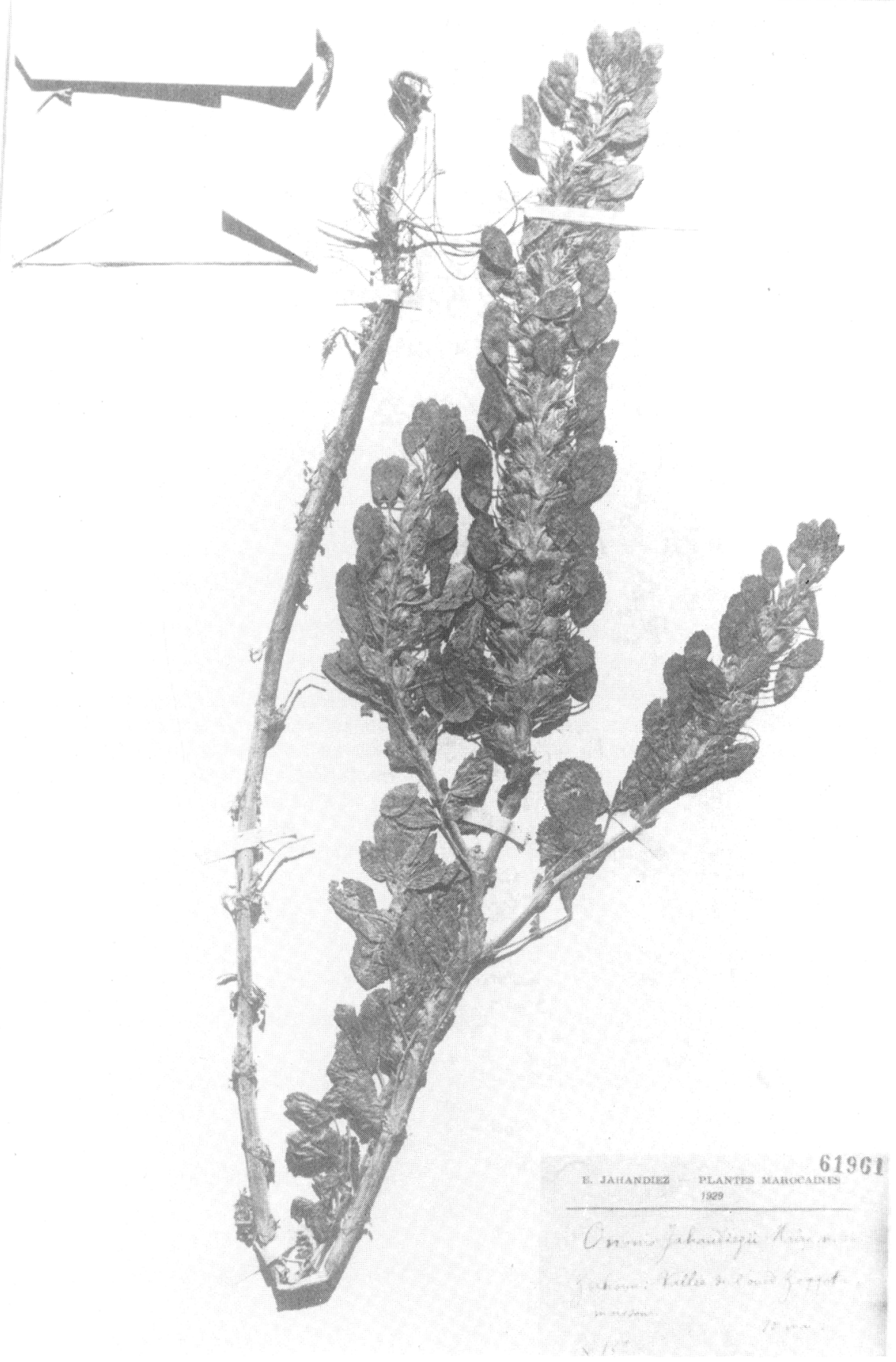

Figura 3.- Ononis jahandiezii Maire \& Weiller. 


\section{BIBLIOGRAFÍA}

DEVESA, J. A. -1986- Ononis sect. Ononis subsect. Diffusae Sirj. en Andalucía Occidental. Lagascalia, 14: 76-85.

DEVESA, J. A. -1988 a-. Estudio taxonómico del género Ononis L. (Leguminosae) en Portugal. Mem. Soc. Brot., 28: 5-23.

DEVESA, J. A. -1988 b- Ononis azcaratei Devesa, sp.nova; Ononis pendula subsp. boissieri (Sirj.) Devesa, comb. et stat.nov.; Ononis viscosa subsp. breviflora var. pitardii Maire; Ononis repens subsp. australis (Sirj.) Devesa, comb. et stat.nov. Lagascalia, 14: 143-145.

DEVESA, J. A. -1992-Sobre la presencia de Ononis euphrasiifolia Desf. en la Península Ibérica. Anales Jard. Bot. Madrid, 50: 118.

GREUTER, W. \& Th. RAUS (Eds.).- 1987- MedChecklist Notulae, 14. Willdenowia 16(2): 439 452.

GREUTER, W., H. M.BURDET \& G.LONG (Eds.) -1989-Med-Checklist, 4. Genève.

IVIMEY-COOK, R. B. -1968- Ononis L. In: T.G.Tutin et al. (Eds.) Flora Europaea, 2: 143148. Cambridge.

JAHANDIEZ, E. y R.MAIRE -1932- Catalogue des Plantes du Maroc, 2. Alger.
LADERO, M. y O. SOCORRO -1982-. Acerca del tratamiento taxonómico de Ononis viscosa $\mathrm{L}$. subsp. crotalarioides (Cosson) Sirj. Stvdia Botanica, 1: 7-10

LÓPEZ, G. -1985- Sobre la identidad de Ononis baetica Clemente. Anales Jard. Bot. Madrid, 42: 250 .

LOSA, T. M. -1958- El género Ononis L. y las Ononis españolas. Anales Inst. Bot. Cavanilles, 26: 227-237.

MAIRE, R. -1931- Contribution à l'étude de la Flore de l'Afrique du Nord, fasc.17. Bull. Soc. Hist. Nat. Afrique. Nord, 22(17): 30-72.

SIRJAEV, G. -1932- Generis Ononis L. revisio critica. Beih. Bot. Centralbl., 49(2): 381-665.

Aceptado para su publicación en Junio de 1996

Dirección del autor. Departamento de Biología y Producción de los Vegetales: Botánica, Facultad de Ciencias, Universidad de Extremadura, Avda. Elvas s/n, E-06071 Badajoz, Spain 